\title{
Viabilidade do Uso da Bolacha-do-mar Mellita quinquiesperforata como Organismo Teste
}

\author{
K. S. Laitano, ${ }^{1 *}$ C. Gonçalves ${ }^{2}$ \& C. Resgalla JR. ${ }^{2 *}$ \\ 1 Labtox/ENS/UFSC, Campus Trindade, Florianópolis, SC \\ ${ }_{2}$ CTTMar/UNIVALI, C.P. 360, CEP 88302-202, Itajaí, SC
}

(Received January 11, 2007; Accepted April 14, 2008)

\begin{abstract}
RESUMO
Este estudo teve por objetivo investigar a viabilidade do uso da bolacha-do-mar Mellita quinquiesperforata como organismoteste em experimentos crônicos de curta duração. Para isto foi aplicado o protocolo da Norma Técnica da CETESB destinada ao ouriço regular Lytechinus variegatus, utilizando embriões e larvas expostos a quatro tóxicos de referência por um período de 24 horas. As substâncias utilizadas foram sulfato de zinco, dicromato de potássio, sulfato de cobre e dodecil sulfato de sódio (DSS). Essas substâncias são normalmente utilizadas para avaliar a sensibilidade relativa de organismos-teste marinhos, assim como para estimar a precisão e confiabilidade dos dados produzidos em laboratório. O protocolo proposto mostrou-se adequado para aplicação com o ouriço irregular M. quinquiesperforata, sendo necessárias algumas modificações quanto às exigências de validação em decorrência da baixa produção de óvulos, baixa taxa de fecundação e maior percentual de efeito observado nos frascos-controle. A CE 50 ;24h média para o metal zinco foi de $50 \propto \mathrm{m} \mathrm{L}^{-1}(\mathrm{CV}$ de $44 \%$ para $\mathrm{n}=5$ ); para o metal cromo, de $1462 \propto \mathrm{g} \mathrm{L}^{-1}$ (CV de 25\% para um $\mathrm{n}$ de 3); metal cobre, de $10 \propto \mathrm{g} \mathrm{L}^{-1}$ (CV de $35 \%$ para $\mathrm{n}=5$ ); e DSS de $2145 \propto \mathrm{g} \mathrm{L}^{-1}(\mathrm{n}=2)$. M. quinquiesperforata apresenta sensibilidade similar às espécies protocoladas pela NBR/ABNT, demonstrando potencial deste Echinodermata para utilização como organismo teste na indisponibilidade das espécies exigidas pelos órgãos ambientais.
\end{abstract}

Palavras-chave: Mellita quinquiesperforata, bolacha-do-mar, teste de toxicidade, zinco, DSS, cromo, cobre.

\section{ABSTRACT}

Viability of the use of the sand dollar Mellita quinquiesperforata as a test organism

The aim of this study was to investigate the viability of the use of sand dollar Mellita quinquiesperforata as a test organism in chronic experiments over short durations. For this, the protocol of the CETESB Technical Standard was used, which was designed for the regular sea urchin Lytechinus variegatus, using embryos and larvae exposed to four reference toxins, for a 24-hour period. The substances used in the toxicity tests were zinc sulfate, potassium dichromate, copper sulfate and sodium dodecyl sulfate (SDS). These substances are normally used to evaluate the relative sensitivity of marine test organisms, and to estimate the precision and reliability of the data produced in the laboratory. The proposed protocol proved to be adequate for its use with the irregular sea urchin M. quinquiesperforata, some modifications being necessary in terms of the validation requirements, due to the low egg production, low fecundation rate, and higher percentage of effect observed in the control flasks. The $\mathrm{EC}_{50} ; 24 \mathrm{~h}$ average for zinc was $50 \propto \mathrm{m} \mathrm{L}^{-1}\left(\mathrm{VC}\right.$ of $44 \%$ for $\mathrm{n}=5$ ), for chrome it was $1462 \propto \mathrm{g} \mathrm{L}^{-1}(\mathrm{VC}$ of $25 \%$ for an $\mathrm{n}$ of 3), for copper it was $10 \propto \mathrm{g} / \mathrm{L}^{-1}$ (VC of $35 \%$ for $\mathrm{n}=5$ ) and for SSD it was $2145 \propto \mathrm{g} \mathrm{L}^{-1}(\mathrm{n}=2)$. M. quinquiesperforata presents a sensitivity similar to species registered by the NBR/ABNT, demonstrating the potential of this Echinodermate for use as a test organism, in cases where it is impossible to use species required by the environmental agencies.

Key word: Mellita quinquiesperforata, sand dollar, toxicity test, zinc, SDS, chromium, copper.

*Corresponding author: Kalinka dos Santos Laitano, e-mail: ka_laitano@yahoo.com.br. 


\section{INTRODUÇÃO}

O ouriço irregular Mellita quinquiesperforata (Leske, 1778) apresenta ampla distribuição geográfica, ocorrendo desde o delta do Mississippi até a Região Sul do Brasil (Borzone, 1993). É uma espécie de grande importância e abundância dentro da comunidade bêntica nas praias arenosas da América do Sul (Borzone et al., 1998). Seu habitat é a zona intermareal com domínio de adultos logo após a faixa de arrebentação, principalmente em praias com granulometria fina a muito fina. Estudos realizados em praias do sul do Brasil demonstraram que a espécie possui ciclo reprodutivo anual, com períodos de desova no final do inverno (agosto/setembro) ou no final do verão (fevereiro/março), dependendo do tipo de praia (Tavares \& Borzone, 1998). Esses autores também destacam que $M$. quinquiesperforata apresenta altas taxas de mortalidade com uma expectativa de vida média de 1 ano (Borzone, 1993), em que organismos de $50 \mathrm{~mm}$ de comprimento teriam idade aproximada de 1 ano para as praias do Paraná e 2 anos para o Rio Grande do Sul (Borzone, 1993; Tavares \& Borzone, 1998).

A utilização de $M$. quinquiesperforata em testes de toxicidade como ferramenta para avaliação de risco e de impacto foi inicialmente proposta pela CETESB (1990), mas não apresentou resultados conclusivos sobre a sua viabilidade. Entretanto, em decorrência da grande extensão da costa brasileira e, conseqüentemente, da variação granulométrica de seu sedimento, a ocorrência de Lytechinus variegatus, espécie protocolada pela CETESB (1992), limita a utilização de testes de toxicidade com Echinodermata a poucos Estados brasileiros.

Segundo o protocolo da USEPA (2002) para testes de toxicidade com organismos marinhos, o uso de espécies nativas que não as citadas no protocolo podem ser estimuladas desde que a sua sensibilidade seja comprovada mediante os resultados obtidos em testes com substâncias de referência.

Os testes com substâncias ou tóxicos de referência são geralmente utilizados para avaliar a sensibilidade relativa de uma população de organismos que será utilizada em testes de toxicidade, permitindo que haja um controle de qualidade sobre a metodologia e também que se estimem a precisão e a confiabilidade dos dados produzidos em laboratório. Nesse tipo de teste, os organismos são expostos a diversas concentrações de uma substância-padrão, sendo que a sensibilidade da população é avaliada através da comparação com dados preeexistentes, disponíveis na bibliografia (Environment Canada, 1992; Resgalla Jr. \& Laitano, 2002).

O objetivo deste trabalho foi investigar a viabilidade do uso da bolacha-do-mar Mellita quinquiesperforata como organismo-teste, verificando a sua sensibilidade e reprodutividade diante de diferentes substâncias de referência em uma adaptação do protocolo destinado para o ouriço regular Lytechinus variegatus. Este estudo pretende fornecer subsídios a diferentes laboratórios cuja localização geográfica impede a realização de testes com Echinodermatas protocolados no Brasil.

\section{MATERIAIS E MÉTODOS}

Para a realização dos testes de toxicidade de desenvolvimento embrio-larval crônico de curta duração de Mellita quinquiesperforata foi adaptado o procedimento apresentado pela Norma Técnica da CETESB de 1992 destinada a testes com Lytechinus variegatus. O método consistiu na obtenção de gametas, fertilização artificial e determinação do número de embriões normais e afetados após um período de exposição de 24 horas.

Para cada bateria de testes foram coletados, no ambiente praial, pelo menos 30 exemplares de adultos da $M$. quinquiesperforata, com diâmetro maior superior a $40 \mathrm{~mm}$ e $5 \mathrm{~mm}$ de altura, para obtenção de um bom volume de óvulos e esperma.

Para a indução da liberação dos gametas foi injetado na região da boca dos organismos $0,5 \mathrm{~mL}$ de cloreto de potássio $(\mathrm{KCl})$ a 1 M. Os óvulos, identificados como grânulos violetaamarelado, foram coletados em vidro-relógio contendo água do mar filtrada. Amostras de óvulos foram então observadas em microscópio para verificar o estágio de maturação e a presença de possíveis anomalias. O líquido espermático, identificado como uma substância branca de consistência leitosa, foi coletado com pipeta de Pasteur. Tanto os óvulos como o esperma, de diferentes indivíduos, foram concentrados em béqueres de $50 \mathrm{~mL}$, sendo os óvulos acondicionados em água do mar filtrada e o esperma concentrado sem diluição sobre o gelo antes do preparo da solução espermática. A solução espermática foi preparada antes da fecundação, que constou na diluição de aproximadamente $200 \mu \mathrm{L}$ do líquido espermático em $10 \mathrm{~mL}$ de água do mar filtrada. Cabe ressaltar que a água do mar utilizada como água de diluição foi coletada no mesmo ambiente onde os adultos de $M$. quinquiesperforata foram coletados. Desta forma, as condições de realização dos testes tornam-se mais próximas à realidade do ambiente natural, pois os organismos já se encontram adaptados às condições físicas e químicas da água.

Para a fecundação foi adicionado de 1 a $2 \mathrm{~mL}$ de solução espermática em um béquer de $250 \mathrm{~mL}$ contendo uma solução de $100 \mathrm{~mL}$ de óvulos e aguardaram-se 10 minutos com agitação constante para a fecundação. Após esse tempo foram observados e quantificados os óvulos fecundados (formação da membrana de fecundação) em amostra diluída em câmara de Sedwick-Rafter. A fecundação mínima determinada para o uso em testes de toxicidade foi de $70 \%$.

Os testes crônicos de curta duração foram executados em seis concentrações da substância, mais o controle em quatro réplicas, em um volume de solução-teste de $10 \mathrm{~mL}$. Todos os experimentos foram realizados a $29 \% \pm 2 \%$, a $25 \pm 2{ }^{\circ} \mathrm{C}$, fotoperíodo de 12 horas escuro e 12 horas de luz durante 24 horas, com observação do efeito do agente químico sobre o desenvolvimento embrionário da espécie. Para a validação do teste foram observados os seguintes requisitos: controles com no mínimo $70 \%$ de organismos bem formados, variação máxima de $20 \%$ da salinidade e pH inicial e final entre 7,5 e 8,5. 
Após o encerramento do teste com adição de $1 \mathrm{~mL}$ de formalina a $4 \%$ observou-se o estágio de desenvolvimento em câmara de Sedgwick-Rafter, registrando o estágio de desenvolvimento e/ou a ocorrência de possíveis anomalias de todos os organismos incubados nos frascos-teste.

Para os testes de toxicidade foram utilizados três tipos de substâncias de referência: dodecil sulfato de sódio (DSS), dicromato de potássio $\left(\mathrm{K}_{2} \mathrm{Cr}_{2} \mathrm{O}_{7}\right)$, sulfato de cobre $\left(\mathrm{CuSO}_{4} .5 \mathrm{H}_{2} \mathrm{O}\right)$ e sulfato de zinco $\left(\mathrm{ZnSO}_{4} .7 \mathrm{H}_{2} \mathrm{O}\right)$ da marca Merck ${ }^{\circledR}$ P.A.

Depois de determinada a porcentagem de efeito foi calculada a CE50 através do método de Trimmed SpermanKarber (Hamilton et al, 1977), utilizando o programa Toxstat 3.4. Uma análise de refração foi realizada para avaliar o número mínimo de organismos a serem quantificados no final do teste. $\mathrm{E}$, finalmente, os resultados obtidos foram comparados com os dados disponíveis para outras espécies de Echinodermatas protocoladas e não protocoladas.

Foram ainda realizados testes de sobrevivência de embriões e larvas da bolacha-do-mar mantidas por 24 horas em sete diferentes salinidades $(5,10,15,20,25,30,35)$ e em quatro réplicas para avaliar sua tolerância e futuras aplicações dos testes de toxicidade com a espécie.

\section{RESULTADOS E DISCUSSÃO}

\section{Variações entre os protocolos de teste com Lytechinus variegatus e Mellita quinquiesperforata}

A aplicação do protocolo de testes embrio-larval do ouriço regular Lytechinus variegatus para a bolacha-do-mar $M$. quiquiesperforata serviu como ponto de partida para a busca de condições de teste mais adequadas para a espécie em questão. De forma geral, protocolos de ensaios ecotoxicológicos são aplicados em diferentes espécies pertecentes à mesma categoria taxonômica, como acontece para bivalvos (His et al., 1999) e Echinodermatas (Clesceri et al., 1998).

Entretanto, algumas diferenças são observadas entre os procedimentos de M. quiquiesperforata e L. variegatus, que podem ser atribuídas a diferenças biológicas intrínsicas de cada espécie.

Para M. quinquiesperforata há a necessidade de coleta e de uso de um maior número de adultos para a realização dos testes, pois a espécie apresenta óvulos de maior tamanho e em menor número que o ouriço L. variegatus. Associado a isto, os percentuais de óvulos não fecundados e de efeito (atraso no desenvolvimento das larvas) nos frascos-controle ao final do experimento são freqüentemente superiores a $20 \%$ e infe-riores a $30 \%$. Esses percentuais são superiores aos observados para $L$. variegatus mas similares aos do ouriço Arbacia lixula (dados não publicados CTTMar/UNIVALI).

Apesar dessas limitações, observou-se que o número mínimo de larvas a serem quantificadas e analisadas após a fixação dos frascos-teste seria de 50 larvas (Figura 1). Esse número foi determinado a partir da estabilização da média e com baixo desvio-padrão mediante a aplicação de uma análise de refração. Este número é a metade exigida para os testes com L. variegatus, amenizando os problemas de limitação de óvulos produzidos, percentual de fecundação e de efeito nos frascos-controle.

As condições gerais para o desenvolvimento do teste de toxicidade utilizando $M$. quinquiesperforata estão sumarizadas na Tabela 1 em comparação com o protocolo de $L$. variegatus. Informações sobre a tolerância e a sensibilidade da bolacha-do-mar em relação à salinidade e temperatura de execução dos experimentos ainda necessitam ser avaliadas. Entretanto, embriões e larvas de M. quinquiespeforata apresentam desenvolvimento normal, ou seja, percentuais de efeito inferior a $30 \%$ em salinidades acima de $25(28,7 \%$ a $25 ; 27 \%$ a 30 e $27,6 \%$ a 35$)$, e $100 \%$ de efeito nas salinidades entre 5 e 20 .

\section{Sensibilidade de Mellita quinquiesperforata diante de outras espécies de Echinodermatas}

Os valores médios de $\mathrm{CE}_{50}$ obtidos nos experimentos com substâncias de referência são apresentados na Tabela 2. Observou-se que os coeficientes de variação (CV) resultantes foram altos $(>30 \%)$ e provavelmente refletem as características da espécie e das condições experimentais executadas neste trabalho.

A partir de estimativas dos valores médios de $\mathrm{CE}_{50} \mathrm{em}$ base da massa atômica dos metais presentes nos sais utilizados nos experimentos, foi possível comparar a sensibilidade de $M$. quinquiesperforata com as demais espécies de Echinodermatas normalmente utilizadas no Brasil (Tabela 3). A bolacha-do-mar apresenta sensibilidade similar às outras espécies, em particular com Lytechinus variegatus e Echinometra lucunter, espécies protocoladas pela ABNT (NBR, 2006). De fato, todas as espécies apresentadas na Tabela 3 apresentam pouca variação de sensibilidade, confirmando o que já vem sendo ressaltado por His et al. (1999), em um estudo de bivalvos, de que a sensibilidade de grupos de espécies de mesma categoria taxonômica apresenta o mesmo potencial de uso em testes de toxicidade. Esta conclusão permitiria o uso de diferentes espécies de Echinodermatas na ausência das espécies protocoladas pelas agências de normatização.

\section{CONCLUSÕES}

Este trabalho apresenta a viabilidade de execução de testes de toxicidade embrio-larval com a bolacha-do-mar Mellita quinquiesperforata. Dos resultados obtidos são sugeridos que:

1. O protocolo dos testes de toxicidade para os ouriços regulares (por exemplo, Lytechinus variegatus) pode ser adaptado para o uso com a bolacha-do-mar.

2. Alguns problemas de uso da espécie em testes de toxicidade foram verificados, como: limitação de 
produção de óvulos e esperma, alto percentual de efeito no controle e alta variabilidade nos resultados.

3. A quantificação do número de larvas no fím do expe-rimento pode ser a metade recomendada pela norma da ABNT, a NBR-15350.

4. É necessário melhor investigação sobre as condições dos experimentos, incluindo análises físicas e quí-micas, tentativas de aumento na taxa de fecundação e redução nos percentuais de efeito observados no controle.
5. Outros estudos deverão ser realizados a fim de reduzir as variabilidades observadas, bem como melhorar as condições de desenvolvimento dos testes.

6. A sensibilidade de $M$. quinquiesperforata é comparável com a de outras espécies de ouriços regulares proto-colados no Brasil.

7. M. quinquiesperforata tem grande potencial para ser utilizado como organismo-teste em testes de toxicidade, em que organismos já protocolados como L variegatus não estão disponíveis.
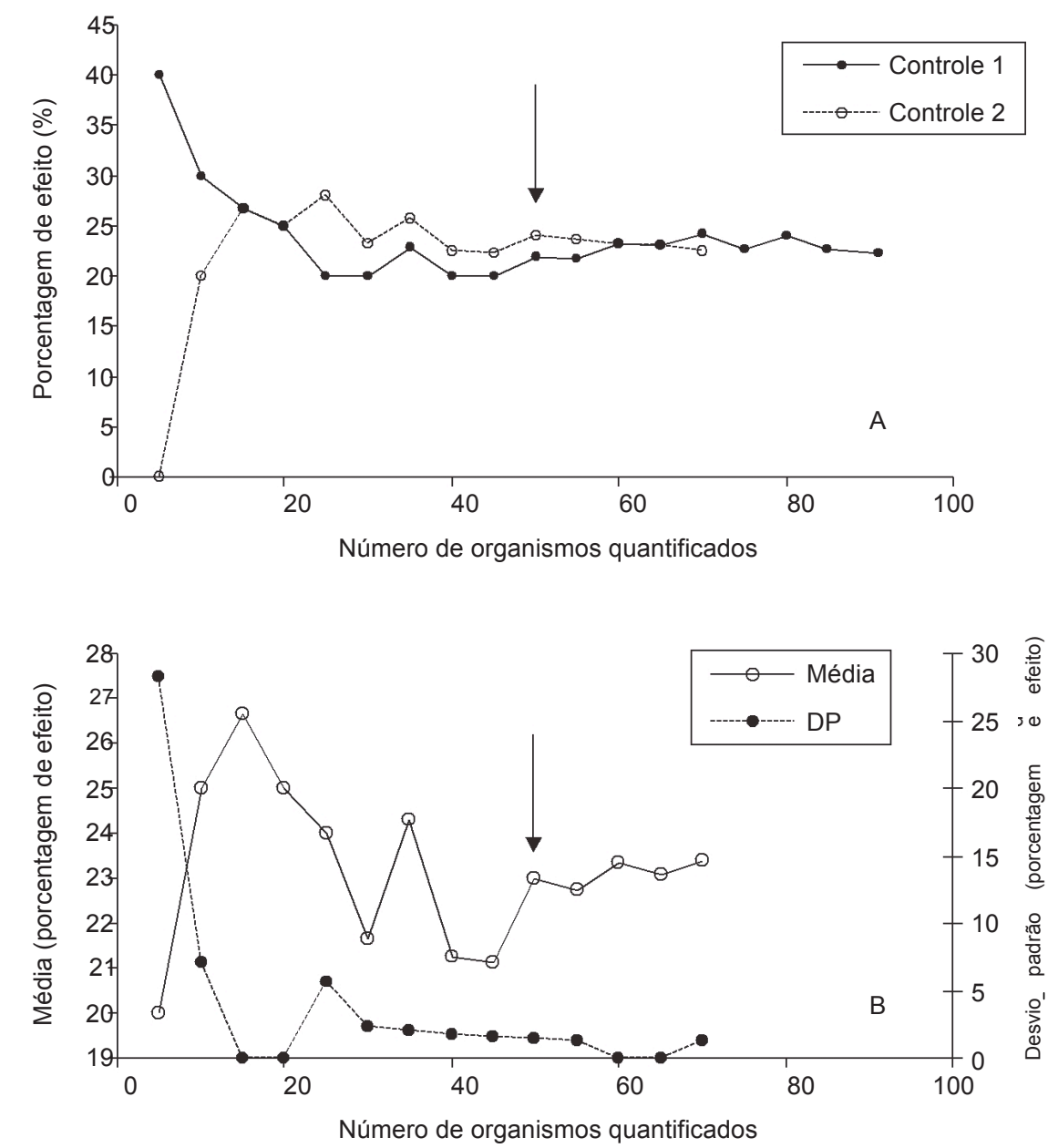

Figura 1 - Análise de refração para determinar o número mínimo de organismos a serem quantificados para os testes de toxicidade com Mellita quinquiesperforata. A - percentual de efeito em dois frascos controle em função do número de organismos quantificados; B - média e desvio-padrão dos percentuais de efeito entre dois frascos-controle em função do número de organismos quantificados. As flechas indicam o número de 50 larvas quantificadas. 
Tabela 1 - Resumo das condições gerais para a realização do teste crônico embrio-larval de curta duração com Mellita quinquiesperforata em comparação com Lytechinus variegatus, de acordo com os procedimentos estabelecidos na norma técnica da CETESB (1992).

\section{Requisitos}

\section{Condições}

\section{Mellita quinquiesperforata Lytechinus variegatus}

Tipo de ensaio

Organismo-teste

Água de diluição

Volume mínimo da solução-teste

№ mínimo de réplicas por diluição

$\mathrm{N}^{\mathrm{o}}$ mínimo de organismos por réplica

Aeração

Alimentação

$\mathrm{pH}$

Salinidade

Temperatura

Fotoperíodo

Duração do teste

Fecundação mínima

Efeito observado

Validade do teste
Estático

Ovos de M. quinquiesperforata Ovos de L. variegatus

Água natural marinha filtrada em filtro menor do que $1 \propto \mathrm{m}$ $10 \mathrm{~mL}$

4

10 ovos $/ \mathrm{mL}$ 30 ovos $/ \mathrm{mL}$

Não

Não

$$
\begin{gathered}
7,5 \text { a } 8,5 \\
29 \pm 2 \\
25 \pm 2{ }^{\circ} \mathrm{C}
\end{gathered}
$$$$
7 \text { a } 9
$$$$
33 \pm 3
$$$$
25 \pm 2{ }^{\circ} \mathrm{C}
$$

$12 \mathrm{~h} \mathrm{luz} \mathrm{e} 12 \mathrm{~h}$ escuro

$16 \mathrm{~h}$ luz e $8 \mathrm{~h}$ escuro

$24 \mathrm{~h}$

$70 \%$

$80 \%$

Retardamento ou anormalidade dos embriões e larvas Mínimo 70\% de larvas pluteus Mínimo $80 \%$ de larvas pluteus normais no controle normais no controle

\begin{tabular}{|c|c|c|c|c|c|}
\hline \multirow{2}{*}{ Substância } & \multirow{2}{*}{$\mathbf{n}$} & \multicolumn{3}{|c|}{$\mathrm{CE}_{50}\left(\mathrm{mg} \mathrm{L}^{-}{ }^{-}\right)$} & \multirow{2}{*}{$\begin{array}{l}\text { CV } \\
(\%)\end{array}$} \\
\hline & & Média & Mínimo & Máximo & \\
\hline Sulfato de zinco & 5 & 0,22 & 0,14 & 0,39 & 44 \\
\hline Dicromato de potássio & 3 & 4,13 & 3,04 & 5,07 & 25 \\
\hline DSS & 2 & 2,14 & 2,09 & 2,2 & - \\
\hline Sulfato de cobre & 5 & 0,04 & 0,03 & 0,07 & 35 \\
\hline
\end{tabular}

Tabela 2 - Número de experimentos (n), valores médios, mínimos e máximos de $\mathrm{CE}_{50} ; 24 \mathrm{~h}$ e coeficiente de variação (CV) obtidos nos testes de sensibilidade de Mellita quinquiesperforata sob efeito dos sais sulfato de zinco, dicromato de potássio, sulfato de cobre e o detergente DSS. 
Tabela 3 - Comparação do valor de CE50;24h de Mellita quinquiesperforata com diferentes espécies de Echinodermatas utilizadas em testes de toxicidade, para os metais zinco, cromo, cobre e detergente DSS.

\begin{tabular}{lccr}
\hline Espécie & Substância & $\propto \mathbf{g ~ L}^{-1}$ & Autor \\
\hline Lytechinus variegatus & & 40,0 & CETESB, 1991 \\
Arbacia lixula & Zinco & 40,0 & Resgalla Jr. \& Laitano (2002) \\
Mellita quinquiesperforata & & $\mathbf{5 0 , 0}$ & Este trabalho \\
Lytechinus variegatus & & 1410,0 & Resgalla Jr. \& Laitano (2002) \\
Arbacia lixula & Cromo & 1470,0 & Resgalla Jr. \& Laitano (2002) \\
Mellita quinquiesperforata & & $\mathbf{1 4 6 2 , 0}$ & Este trabalho \\
Arbacia lixula & & 1240,0 & Resgalla Jr. \& Laitano (2002) \\
Lytechinus variegatus & & 2370,0 & Rumbold \& Snedaker (1997) \\
Echinometra lucunter & DSS & 3000,0 & Araujo \& Nascimento (1999) \\
Mellita quinquiesperforata & & $\mathbf{2 1 4 5 , 0}$ & Este trabalho \\
Arbacia lixula & & 46,0 & Resgalla Jr. \& Laitano (2002) \\
Echinometra lucunter & Cobre & 21,9 & Rumbold \& Snedaker (1997) \\
Lytechinus variegatus & & 33,8 & Rumbold \& Snedaker (1997) \\
Mellita quinquiesperforata & & $\mathbf{1 0 , 0}$ & Este trabalho \\
\hline
\end{tabular}

\section{REFERÊNCIAS BIBLIOGRÁFICAS}

ARAÚJO, M. M. S. \& NASCIMENTO, I. A., 1999, Testes ecotoxicológicos marinhos: análise de sensibilidade. Ecotox. Envir. Rest., 2: 41-47.

BORZONE, C. A., 1993, Spatial distribution and growth of Mellita quinquiesperforata (Leske, 1778) on a sandy beach of southern Brazil. Nerítica, 7: 87-100.

BORZONE, C. A., TAVARES, Y. A. G. \& BARROS Jr., F. C. R., 1998, Beach morphodynamics and distribution of Mellita quinquiesperforata (Leske, 1778) on sandy beaches of southern Brazil. In: Mooi \& Telford (eds.), Echinoderms: San Francisco. Balkema, Rotterdam, pp. 581-586.

CETESB, 1990, Desenvolvimento e implantação de testes de toxicidade com organismos marinhos. Relatório, 83.05.00. 38p.

CETESB, 1991, Desenvolvimento e implantação de testes de toxicidade com organismos aquáticos - Testes com organismos marinhos. Relatório, volume 3, 58.02.00, 26p.

CETESB, 1992, Água do mar - Teste de toxicidade crônica de curta duração com Lytechinus variegatus, Lamark, 1816 (Echinodermata:Echinoidea). Norma Técnica L5.250, 20p.

CLESCERI, L. S., GREENBERG, A. E. \& EATON, A. D., 1998, Standard methods for the examination of water and wastewater. 20. Edition, Washington, D.C., American Public Health Association.

ENVIRONMENT CANADA, 1992, Biological test method: Acute test for sediment toxicity using marine or estuarine amphipods. Report EPS1/ $\mathrm{RM} / 26$.
HAMILTON, M. A. A, RUSSO, R. C. \& THURSTON, R. V., 1977, Trimmed Sperman-karber method for esttimatring median lethal concentrations on toxicity bioassays. Environ. Sci. Technol., 11: 714719.

HIS, E., BEIRAS, R. \& SEAMAN, M. N. L., 1999, The assessment of marine pollution - bioassays with bivalve embryos and larvae. $A d v$. Mar. Biol., 37: 1-178.

NBR, 2006, Ecotoxicologia aquática - Toxicidade crônica de curta duração - Método de ensaio com ouriço-do-mar (Echinodermata: Echinoidea). NBR-15350, 17p.

RESGALlA Jr., C. \& LAITANO, K. S., 2002, Sensibilidade dos organismos marinhos utilizados em testes de toxicidade no Brasil. Notas Téc. Facimar, 6: 153-163.

RUMBOLD, D. G. \& SNEDAKER, S. C., 1997, Evaluation of bioassays to monitor surface microlayer toxicity in tropical marine waters. Arch. Environ. Contam. Toxicol., 32: 135-140.

TAVARES, Y. A. G. \& BORZONE, C. A., 1998, General features of population dynamics of the sand dollar Mellita quinquiesperforata (Leske, 1778) in southern Brazilian sandy beaches. In: Mooi \& Telford (eds.), Echinoderms: San Francisco. Balkema, Rotterdam, pp. 837-842.

USEPA, 2002, Short-term methods for estimating the chronic toxicity of effluents and receiving waters to marine and estuarine organisms. 3. Edition, Washington, EPA-821-R-02-014. 